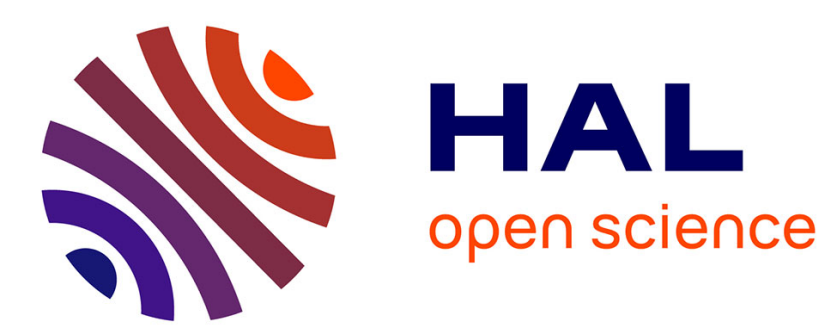

\title{
Le digital labor : humain, trop humain
}

Frédérique Pallez

\section{To cite this version:}

Frédérique Pallez. Le digital labor: humain, trop humain. Gérer et Comprendre. Annales des Mines, 2019, N¹38 (4), pp.61-62. 10.3917/geco1.138.0061 . hal-02444129

HAL Id: hal-02444129

https://hal-mines-paristech.archives-ouvertes.fr/hal-02444129

Submitted on 17 Jan 2020

HAL is a multi-disciplinary open access archive for the deposit and dissemination of scientific research documents, whether they are published or not. The documents may come from teaching and research institutions in France or abroad, or from public or private research centers.
L'archive ouverte pluridisciplinaire HAL, est destinée au dépôt et à la diffusion de documents scientifiques de niveau recherche, publiés ou non, émanant des établissements d'enseignement et de recherche français ou étrangers, des laboratoires publics ou privés. 


\title{
Frédérique PALLEZ \\ Le digital labor : humain, trop humain
}

\author{
À propos de l'essai d'Antonio CASILLI, En attendant les robots. Enquête sur le travail du \\ clic, Le Seuil, coll. « La couleur des idées », 2019
}

A paraître dans Gérer \& Comprendre, 2019/4

L'intelligence artificielle est-elle l'avenir de l'humanité ? En tout cas, l'engouement qu'elle suscite masque une réalité qu'Antonio Casilli nous dévoile dans une enquête détaillée et passionnante : derrière le mythe d'activités entièrement automatisées et la peur de la « disparition du travail » se cachent en fait de nouvelles formes de travail, bien humain celuici, le digital labor, qui métamorphose le geste productif humain en une multitude de microopérations, sous-payées ou même non payées, nécessaires au traitement des données qui alimentent la nouvelle économie informationnelle que nous connaissons. Cette transformation du travail est inséparable du développement des plateformes.

2L'ouvrage est organisé en trois parties. La première (Quelle automation ?) analyse le lien entre le programme de l'intelligence artificielle et le paradigme des plateformes; la deuxième décortique les formes que prend le digital labor à partir d'exemples; la troisième cherche à penser théoriquement les phénomènes de surexploitation des individus et d'asymétrie économique mis au jour, pour fournir quelques pistes permettant de les dépasser.

3Dans la première partie, l'auteur réexamine le débat sur la possible disparition du travail humain, en nous montrant que c'est surtout sur un plan qualitatif plus que quantitatif que l'automation fait sentir ses effets : elle aboutit à une standardisation et une externalisation des tâches, par ailleurs de plus en plus fragmentées. Des milliards de petites mains, selon l'auteur, bien loin des experts informaticiens que l'on imagine, interviennent quotidiennement, parfois à leur insu (comme vous et moi), pour assister, maintenir, contrôler, entraîner des machines qui ne peuvent fonctionner sans elles. Et les plateformes, formes hybrides entre entreprise et marché, captent ainsi la valeur produite par les mécanismes de coordination multiface qu'elles organisent entre les divers types d'utilisateurs. Ceux-ci contribuent à cette création de valeur sous trois formes : le travail à la demande, le micro-travail, et le travail social en réseau.

$\underline{4}$ Ces trois formes sont analysées successivement dans la deuxième partie, qui en démonte les mécanismes, exemples connus de tous à l'appui. La plateforme Uber, qui illustre le premier type, le travail à la demande, met ainsi au travail non seulement les chauffeurs (qui font beaucoup plus que conduire leur véhicule) mais aussi les usagers, qui, chacun de leur côté, se livrent à un intense travail de qualification (notation des chauffeurs, renseignement de profils...), de production de données monétisables (temps de trajet, par exemple) qui seront réutilisés par la plateforme.

5Le micro travail correspond, lui, à des activités paradoxalement simples, comme l'annotation de vidéos, le tri d'adresses, le contrôle de documents..., que les machines ne peuvent effectuer efficacement elles-mêmes. La plateforme de micro-travail d'Amazon, Mechanical Turk, en est une illustration frappante : elle met en relation une entreprise « requérante », cherchant par exemple à trier des milliers de pages d'archives manuscrites, et 
500000 micro-travailleurs, qui en retranscriront deux lignes chacun. La rémunération de ce type de tâches, à hauteur de quelques centimes, est un revenu complémentaire pour un certain nombre d'Américains (essentiellement des femmes) qui les effectuent, mais, hors des EtatsUnis, la ressource principale d'un foyer pour les $20 \%$ de travailleurs (indiens par exemple) qui y contribuent. S'agit-il de travail ? La plateforme se présente comme intermédiaire entre requérant et travailleurs (et refuse la position d'employeur) et s'emploie à mettre « hors travail » ces activités, par exemple en mettant en place des procédures de « gamification » et d'émulation, qui sont en fait des mises en concurrence et des contrôles dissimulés. Non contente de se faire rémunérer par les entreprises requérantes (ses clients), elle utilise également les données de qualité produites par les travailleurs du clic pour « former » les machines, par exemple les algorithmes de traduction automatique. Les «microtâcherons » inscrivent ainsi en permanence de l'intelligence humaine au sein des logiciels, et sont au cœur des stratégies de big data de toutes les grandes plateformes. Mais doivent-ils être considérés comme des freelances ou des galériens du clic ? En fait, cette question est plus complexe qu'il n'y paraît car, derrière quelques « freelances » qui gagnent correctement leur vie, se dissimulent des sous-traitants à qui ceux-là ont délégué des microtâches..., tout ceci s'effectuant le cas échéant sur des continents différents, et venant parachever un modèle de la délocalisation déjà bien installé. Cerise sur le gâteau, dans un certain nombre de cas, le travail n'est pas rémunéré, et les usagers font l'objet d'une demande explicite de participation à l'amélioration de la plateforme sous couvert de jeu, usagers qui en outre se contrôlent mutuellement sur le travail effectué... C'est là que le travail des «microtâcherons » se métamorphose en la troisième forme identifiée par l'auteur, le « travail social en réseau ».

6Dans cette troisième catégorie de travail, même s'il se contente de "liker" une page, l'usager devient «produsager » c'est-à-dire producteur de contenu et, en même temps, animateur d'une communauté qu'il contribue à fabriquer. On peut alors se demander s'il s'agit d'un mécanisme insidieux d'exploitation de l'usager par les plateformes ou si celui-ci, en amateur connecté, hédoniste, contribue, comme le défendent certains chercheurs, à développer une intelligence sociale plus démocratique et ouverte. Le débat fait rage entre les deux visions, mais pour l'auteur, il faut d'abord prendre conscience que l'usager « actif » ne représente qu'une faible minorité (de l'ordre de $1 \%$ ), que les plateformes ont d'abord cherché à encourager, y compris pécuniairement, avant de se rendre compte qu'en brouillant ainsi la frontière entre bénévoles et professionnels, elles risquaient un retour de bâton. C'est effectivement ce qui s'est passé, et l'auteur nous narre différentes actions organisées par ces «produsagers » avec des succès divers, pour faire reconnaître leur travail et le faire rémunérer. En fait, on s'aperçoit que le travail « gratuit » n'est pas si répandu sur les plateformes, tant sont nombreux les mécanismes qui distinguent plusieurs groupes d'usagers, qui ne sont pas soumis aux mêmes règles de tarification. En outre, l'auteur montre que ce n'est pas tant la production de contenu qui constitue la valeur produite, que les profils personnels et les interactions réalisées par les internautes, contribuant aux mécanismes de qualification, qui alimenteront les data dont sont friandes les grandes plateformes, ou qui pourront être monétisées directement (on peut s'acheter des fans ou des followers sur les plateformes sociales...).

7La description minutieuse de ces fonctionnements, dont la complexité ne permet de donner ici qu'un faible aperçu, conduit ensuite l'auteur à poser des questions plus générales relatives à la nature et à l'avenir de ce digital labor, en se positionnant par rapport à différents auteurs de l'économie et de la sociologie. Ce que nous montre finalement Antonio Casilli, comme le note la sociologue Dominique Méda dans une postface éclairante, c'est l'émergence d'une nouvelle forme de travail, qui fait exploser les repères habituels : travail ô combien matériel 
dans une économie qui se dit immatérielle, travail dont les frontières avec le jeu se brouillent souvent, travail occulté qui exige de l'invisibilité pour produire ce qui passe pour de l'automation, travail qui remplace la relation de subordination à un employeur par une multitude de dépendances sans limites temporelles ni spatiales, travail dont la rémunération, pouvant aller jusqu'à la gratuité, ne constitue plus une caractéristique essentielle. L'auteur débouche alors sur le constat d'une aliénation progressive des travailleurs du clic, contre laquelle il examine, pour terminer, deux voies possibles de résistance : l'une s'attache à élargir au digital labor les conquêtes sociales liées à l'emploi formel ; l'autre cherche à renouer avec l'inspiration initiale des plateformes pour refonder, sur la base de la théorie des communs, un nouveau rapport entre l'usager-producteur et les infrastructures de traitement des données. Mais il s'agit de pistes, dont le développement reste à mener, et, au bout du compte, de véritables programmes politiques.

$\underline{8}$ Cet ouvrage invite, comme le lecteur l'aura compris, à passer derrière le rideau des propos apocalyptiques ou lénifiants qui pullulent actuellement sur « la plateformisation », l'uberisation, l'intelligence artificielle, les bienfaits ou les risques du « numérique »... Antonio Casilli nous emmène au contraire dans l'arrière-cuisine : l'habitacle du chauffeur Uber, le père de famille indien qui annote des images en série, les fermes à clic qui gonflent sur commande la notoriété d'un homme politique, mais aussi vous et moi quand nous postons des photos de voyage ou «partageons » des opinions... Son enquête très précise, menée pendant deux ans, utilise le fil conducteur de la notion de travail pour démonter des mécanismes de fonctionnement d'une grande opacité, mais aussi d'une inventivité et d'une variété confondantes, qui sont les moteurs de cette nouvelle économie numérique dans laquelle nous baignons chaque jour davantage. A ce titre, la lecture de son livre est de salubrité publique, pour, au moins pendant un moment, tenter de faire un pas de côté et comprendre ce à quoi nous participons tous à des degrés divers, comme travailleurs du clic «sans le savoir ». 